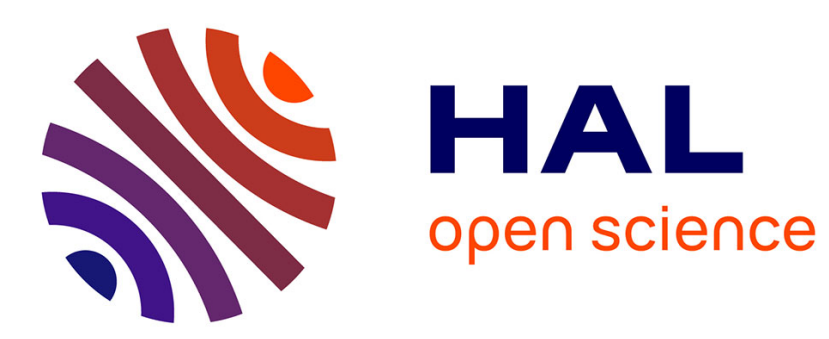

\title{
A novel single-ended fault identification algorithm for full selective protection strategy
}

Paul Verrax, Alberto Bertinato, Michel Kieffer, Bertrand Raison

\section{To cite this version:}

Paul Verrax, Alberto Bertinato, Michel Kieffer, Bertrand Raison. A novel single-ended fault identification algorithm for full selective protection strategy. 15th International Conference on Developments in Power System Protection (DPSP 2020), Mar 2020, Liverpool, United Kingdom. 010 (6 pp.)-010 (6 pp.), 10.1049/cp.2020.0059 . hal-03097231

\section{HAL Id: hal-03097231 \\ https://hal-centralesupelec.archives-ouvertes.fr/hal-03097231}

Submitted on 5 Jan 2021

HAL is a multi-disciplinary open access archive for the deposit and dissemination of scientific research documents, whether they are published or not. The documents may come from teaching and research institutions in France or abroad, or from public or private research centers.
L'archive ouverte pluridisciplinaire HAL, est destinée au dépôt et à la diffusion de documents scientifiques de niveau recherche, publiés ou non, émanant des établissements d'enseignement et de recherche français ou étrangers, des laboratoires publics ou privés. 


\title{
A novel single-ended fault identification algorithm for full selective protection strategy
}

\author{
Paul Verrax ${ }^{*}$, Alberto Bertinato*, Michel Kieffer ${ }^{\dagger}$, Bertrand Raison $^{\ddagger}$ \\ * Supergrid Institute, 23 rue Cyprian, 69100 Villeurbanne \\ ${ }^{\dagger}$ L2S, CNRS-CentraleSupelec-Univ Paris-Sud, Univ Paris-Saclay, 3 rue Joliot-Curie, 91192 Gif-sur-Yvette. \\ ${ }_{\ddagger}^{\ddagger}$ Univ. Grenoble Alpes, CNRS, Grenoble INP*, G2Elab, 38000 Grenoble, France (*Institute of Engineering Univ. Grenoble Alpes)
}

Keywords: HVDC, Multi-terminal, protection, fault detection, fault localization, parameter identification.

\begin{abstract}
While more and more HVDC point-to-point connections are initiated worldwide, the emergence of multi-terminal grids still faces important hurdles among which the protection is seen as particularly challenging. We propose a parametric single-ended fault identification algorithm to be used in fullyselective fault-clearing strategies. Such strategies are preferential for meshed grids as they limit the impact of the fault. The method is able to determine whether the line monitored by a relay is faulty or not using very few local measurements, making ultra-fast fault identification possible. Contrary to most existing approaches, extra inductances are not required to distinguish between faults occurring on the protected line and elsewhere. When a fault is suspected, the proposed algorithm estimates the parameters that characterize the fault, namely the fault distance and impedance. The estimation process uses a parametric model describing the voltage and current evolution just after the fault occurrence based on the fault parameters. The identification of the faulty line is then based on the size of the confidence region of the estimated fault parameters. Use-cases and performances of the algorithm for a fournode meshed grid are studied using Electro-Magnetic Transient (EMT) simulations.
\end{abstract}

\section{Introduction}

Multi-Terminal high-voltage Direct-Current (MTDC) grids have recently been the subject of many studies. In the future, such type of grids is likely to act as a backbone to the existing AC network, providing better interconnection over large distances between renewable energy sources and consumption area as well as integration of large offshore wind power plants [1]. Among the several technical and non-technical barriers still to overcome for the development of High-Voltage DirectCurrent (HVDC) meshed grids, the protection of the lines is seen as one of the most challenging [2]. Protection strategies and breaker technologies developed in the case of High Voltage Alternative Current grids cannot be directly translated to
HVDC grids, since, for example, faults on an HVDC line, do not lead to a zero-crossing of the current, which makes the fault clearance more difficult.

The main tasks of a protection strategy $\mathrm{t}$ include, but is not limited to, fault detection, faulty component identification, and tripping of the breakers [3]. In a full selective fault clearing strategy, the faulty component is identified prior to the fault clearing so that only the faulty component is disconnected, which is considered preferable since this minimizes the impact of the fault on the grid. In such a strategy, Direct Current Circuit Breakers (DCCB) located at the end of each line are responsible for the protection of their respective line, see Figure 1. Overall, the protection strategy must be reliable, i.e., it must lead to an isolation of the protected line only when needed and must be immune to changes caused by normal operations on the grid. Finally, the protection algorithm must operate fast enough to cope with the DCCB capabilities, typically in less than a millisecond, which makes use of communication between distant protection devices not suitable. Hence, a selective protection scheme requires a single-ended (non-unit) algorithm [3] ensuring selectivity, i.e., able to discriminate between internal faults, occurring on the protected line and external faults, occurring elsewhere in the grid.

A faulty behavior is generally associated with high variation rates in both current and voltage. Various measurements-based methods using thresholds on voltage and their derivatives [4] or current and their derivatives [5], or both, have been proposed. They all benefit from costly inductances placed at the end of each line, between the relay and the bus-bar, see Figure 1.

Model-based methods try to benefit from a more accurate description of the evolution of current and voltage when a fault appears on a line. In the context of AC transmission multiple traveling wave arrival times are considered in [6] to spot the reflection patterns between the observation point and the fault. Several wave arrival times (about a dozen) need to be acquired, which limits the speed of the method. The concept of distance protection is extended to the HVDC case in [7]. Considering a frequency dependent model applied in time domain, the fault distance is computed and compared with some setting distance, which is used as criterion for fault detection. However, the measurements must be observed during more than $10 \mathrm{~ms}$ to 
accurately locate the fault and the method is not tested against faults that do not occur on the protected line.

Model-based algorithms are hence considered for fault detection and identification. Nevertheless, finding a good trade-off between quick detection and identification and accurate (therefore complex) estimation of the fault characteristics is still challenging.

This paper introduces a novel single-ended protection algorithm able to detect and identify faults occurring on transmission lines. Each relay embeds a parametric model of the evolution of the current and voltage in case of a fault, depending on a set of physical fault parameters. When a fault is suspected, an iterative maximum-likelihood (ML) estimate of the fault parameters is evaluated from the data available at the relay. The estimated fault parameters and their confidence intervals are exploited to determine whether or not one has to consider that a fault actually occurred on the protected line. The motivation and benefits of a fast fault clearing strategy is detailed in Section 2. The proposed fault identification algorithm is presented in 3.1. Simulation results using EMT software [8] are presented in Section 4.

\section{Motivation and algorithm use cases}

In this section we justify the feasibility and benefit of a full selective fault clearing strategy using the proposed ultra-fast fault identification algorithm. Thus we consider here that a single-ended algorithm able to detect and identify faults in $0.5 \mathrm{~ms}$ is implemented at each relay, which is motivated in Section 4. The test grid is first introduced in Section 2.1. Then we will show that in the context of Over-head line (OHL) based MTDC, the combination of fast fault identification and Hybrid DCCB makes it possible to selectively clear the fault without considering extra DC inductances, see Section 2.2. In Section 2.3 we will focus on the possibility to prevent converter stations from auto-blocking due to the high fault currents. In those sections, the simulations are conducted without assuming any action from the breakers.

\subsection{Test grid}

The considered grid is a four station meshed HVDC grid as introduced in the PROMOTIoN project [3], adapted with overhead lines. Note that DC inductances are omitted. Modular Multilevel Converter (MMC) stations have a rated power of $1 \mathrm{GW}$. An asymmetrical monopole with single conductor configuration is considered. In the followings we will consider different fault cases occurring on line $\mathrm{L}_{14}$ with various fault distances $d_{\mathrm{f}}$ and fault resistances $R_{\mathrm{f}}$.

\subsection{Coping with the rise of current}

Short circuits due to faults in high voltage transmission lines induce important currents along the faulted line. If one aims at clearing only the faulted line, the line breakers must trip before

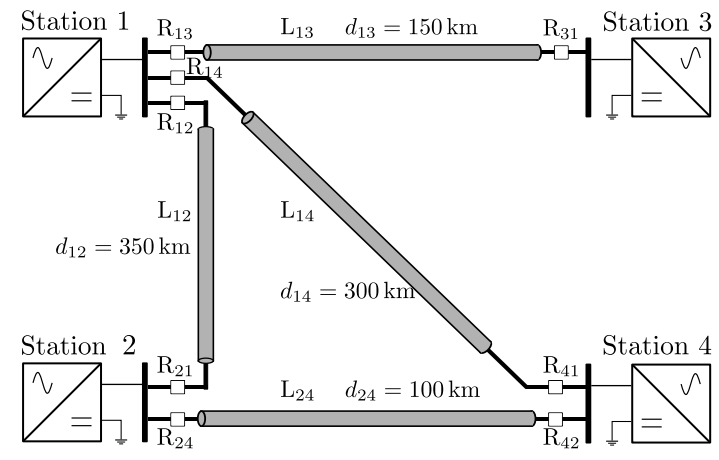

Fig. 1: Four station test grid where an asymmetrical monopole single conductor configuration with earth return is considered

the current rises above their breaking capability. In the case of underground cable based grids, the current rises extremely fast, making the presence of smoothing reactors necessary at the DC side of the converter or in series with the breakers. However, for OHL based grids, it may be possible to remove these inductances because of the inductive behavior of the line that attenuates the rise of the current. This is beneficial due to the important cost of such inductances and the negative impact they could have on the dynamics of the grid [9]. However, the current may still reach high values depending on the position and impedance of the fault.

The use of hybrid circuit breakers is seen as a good compromise between opening time, one-line losses and breaking capabilities. The considered DCCB have a breaking capabilities of $16 \mathrm{kAand}$ an operating time of $2 \mathrm{~ms}$ [10]. Added to the time required for the fault identification, the total fault neutralization time is considered to be $2.5 \mathrm{~ms}$. Thus, to validate the possibility to disconnect only the faulted line one has to check if the current rises above $16 \mathrm{kA}$ in less than $2.5 \mathrm{~ms}$ after the fault reaches a given relay. The Figure 2 shows the value of the line current for a fault on the line $\mathrm{L}_{14}$, for different values of fault distance and resistance. The $16 \mathrm{kA}$ maximum breaking capability of the breakers is also displayed. For all the fault cases the level of the current allows to use the breakers at the relay and disconnect the faulted line. The use of a fast faultidentification algorithm hence enables the recourse to a fullselective fault clearing strategy while omitting the smoothing reactors on the DC side.

\subsection{Preventing the stations blocking}

During a fault the current flowing through the MMC stations will rise and eventually triggers the power electronic components self-protection. The IGBTs will be blocked if the current of the arm reaches a certain threshold, usually considered to be 2 p.u. of the rated current [11]. This has a negative impact on the healthy lines connected to the blocked stations as it prevents the station to control the power flow on those lines. After a fault is cleared the station must hence be de-blocked to return to normal operation. However the fast opening of the line 

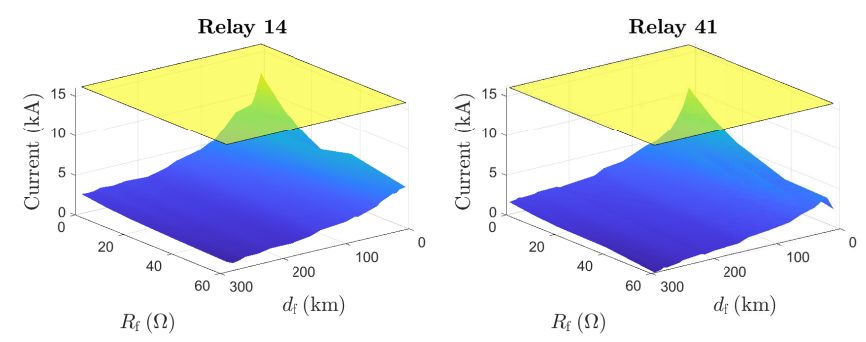

Fig. 2: Line current after $2.5 \mathrm{~ms}$ at relays $\mathrm{R}_{14}$ and $\mathrm{R}_{41}$ when a fault occurs on line $L_{14}$. Fault distances are taken with respect to the considered relay, i.e., from relay $\mathrm{R}_{14}$ (left) and $\mathrm{R}_{41}$ (right).

circuit breakers can prevent the station from blocking. This would diminish the fault impact on the grid and decrease the total duration of the protection strategy [12].

Here we investigate for which fault cases does the proposed protection strategy allow to avoid the blocking of the MMC stations. To do so we consider the time instant at which the converter at station $i$ blocks $t_{\text {block }}^{(i)}$, taken with respect to the fault arrival time at station $i$. The blocking instants at stations 1 and 4 are displayed in Figure 3 for the different fault cases occurring on line $\mathrm{L}_{14}$.If $t_{\text {block }}^{(i)} \geq 2.5 \mathrm{~ms}$ the opening of the DCCB on the faulted line would prevent the station from blocking. The behavior of the other stations not directly connected to the faulted line is not showed as the time scales involved are less constraining. The beige area corresponds to fault cases for which the blocking of the stations is too fast for the DCCB to prevent it, i.e., $t_{\text {block }}^{(i)} \leq 2.5 \mathrm{~ms}$, the blue area depicts the faults for which the station blocks more than $30 \mathrm{~ms}$ after the fault inception at the station, or may never block. In between, the green area corresponds to the cases where the blocking of the station would be avoided by clearing the the fault in $2.5 \mathrm{~ms}$. The latter concerns mostly low impedance faults whose distance from the considered relay can go up to the total length of the line. Furthermore it is noticeable that even in the worst cases only one converter station blocks, as for instance close faults as seen from station 1 are remote faults for station 4 . This is an expected requirement in meshed HVDC grids [13]. The proposed strategy can thus increase the availability of the grid for a wide range of fault cases by preventing the stations from blocking.

\section{Problem formulation and proposed approach}

\subsection{Problem statement}

Consider a fault occurring at some time instant $t_{\mathrm{f}}$ in the grid on a line $e$ of length $d_{q q^{\prime}}$ connecting stations $q$ and $q^{\prime}$. The distances between the fault and the stations $q$ and $q^{\prime}$ are $d_{\mathrm{f}, q}$ and $d_{\mathrm{f}, q^{\prime}}=d_{q q^{\prime}}-d_{\mathrm{f}, q}$. The fault is assumed to be characterized by its pole-to-ground impedance $Z_{\mathrm{f}}$, considered constant during the time interval of interest in the order of a millisecond [14]. The vector of parameters describing a fault is
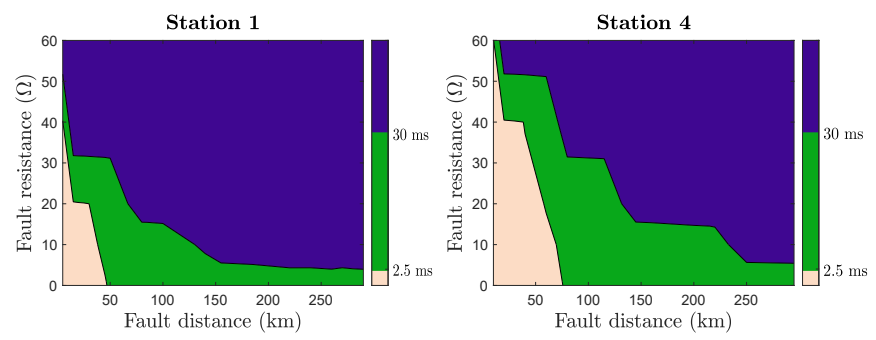

Fig. 3: Time instant at which the stations 1 and 4 block for faults occurring on line $\mathrm{L}_{14}$. Fault distances are taken with respect to the considered station, i.e., from station 1 (left) and 4 (right).

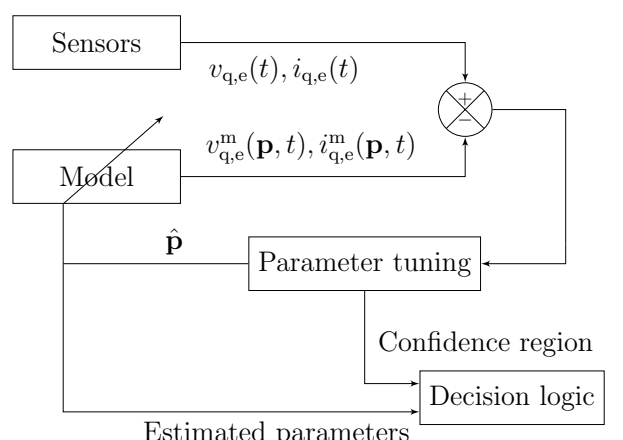

Fig. 4: Overview of the proposed fault identification approach.

thus $\mathbf{p}=\left(e, d_{\mathrm{f}, q}, Z_{\mathrm{f}}, t_{\mathrm{f}}\right)^{\mathrm{T}}$. During the parameter estimation process the line $e$ is fixed. Furthermore $d_{\mathrm{f}, q}$ and $t_{\mathrm{f}}$ are linked through the detection time of the first traveling wave at station $q, t_{\mathrm{d}, q}=t_{\mathrm{f}}+\frac{d_{\mathrm{f}, q}}{c_{\mathrm{w}}}$, which can be measured. $c_{\mathrm{w}}$ is the propagation speed of the traveling waves, determined by the line parameters. Thus the vector of the fault parameters is reduced to $\mathbf{p}=\left(d_{\mathrm{f}, q}, Z_{\mathrm{f}}\right)^{\mathrm{T}}$.

Assume that the Fault Identification Device (FID) of station $q$ monitoring line $e$ acquires at a frequency $f$ voltage and current measurements $\left(v_{q, e}(t), i_{q, e}(t)\right)$ at the end of $e$ connected to $q$. Using, e.g., an under-voltage criterion [15], the FID is able to determine whether the grid behaves normally or not.

Once an abnormal behavior has been detected, the FID has to determine whether or not the fault occurred in the monitored line. The proposed approach, see Figure 4, considers a parametric model of the evolution of voltage and current at node $q$ in case of a fault, detailed in Section 3.2. The fault parameter tuning process and the decision logic are presented Section 3.3.

\subsection{Fault modeling}

\subsubsection{Physical modeling}

After the occurrence of a fault, the transient behavior of current and voltage measured at a given point of a line $e$ connecting stations $q$ and $q^{\prime}$ can be modeled using traveling waves [16]. 
The telegraph equations allow one to represent the propagation and attenuation of the voltage and current waves as they travel along the line as

$$
\frac{\partial^{2} V}{\partial x^{2}}=Z(s) Y(s) V(x, s)
$$

where $Z(s)=R+s L$ and $Y(s)=G+s C$ are the transfer functions of the distributed series impedance and shunt admittance, respectively. For high voltage over-head lines, a loss-less approximation is relatively accurate so that only the per length inductance $L$ and capacitance $C$ can be considered. The latter corresponds to the line to earth impedance and does not vary significantly with the frequency. However, $L$ represents both the self inductance of the line as well as the inductance due to the magnetic field in the earth. The latter depends greatly upon the frequency and the nature of the ground, represented by the soil resistivity $\rho$. In the first stage of our approach, we neglect this effect due to the soil resistivity. Setting $\rho=0$ allows us to analytically solve the Telegraph equations 1 in the Laplace domain with parameters $L, C$ fixed at a given frequency.

The fault may be represented by a voltage source in series with an impedance $Z_{\mathrm{f}}$ between the ground and the fault location connected at the fault instant $t_{\mathrm{f}}$. The fault impedance accounts for different effects such as the electric arc, the tower grounding impedance, and the resistance of additional unknown objects in the current path. Here, as in [14], these effects are described considering a single unknown fault resistance, i.e., $Z_{\mathrm{f}}=R_{\mathrm{f}}$. The converter stations, here MMC, are modeled by an RLC equivalent. The voltage and current at the station $q$ located at the end of the line $e$ is the sum of the different incident and reflected traveling waves at this station. Such expressions can be explicitly derived in time domain by taking the inverse Laplace transform and depend on known characteristics of the grid, and on measured quantities such as the detection time as well as the voltage and current just before the occurrence of the fault. These expressions also depend on the fault parameter vector $\mathbf{p}=\left(d_{\mathrm{f}, q}, R_{\mathrm{f}}\right)^{\mathrm{T}}$, which is unknown.

\subsubsection{Accounting for the soil resistivity}

The physical model introduced previously neglects the effect of the soil resistivity. Here, we assume the soil resistivity can be represented by a known constant parameter $\rho$ along the return path of the monitored line. To account for soil resistivity effects, we supplement the physical model with a behavioral model, to get a combined model, see Figure 5. Consider the output $y^{\mathrm{m}, 0}(\mathbf{p}, t)=\left(v^{\mathrm{m}, 0}(t, \mathbf{p})^{T}, i^{\mathrm{m}, 0}(t, \mathbf{p})^{T}\right)^{T}$ of the physical model representing the voltage and current at a given point of the monitored line $e$. Preliminary simulations have shown that the soil resistivity impacts the model output $y^{\mathrm{m}, 0}(\mathbf{p}, t)$ as a low-pass filter. Consequently, we choose to describe the output $y^{\mathrm{m}, \mathrm{g}}=\left(v^{\mathrm{m}, \mathrm{g}}(t, \mathbf{p})^{T}, i^{\mathrm{m}, \mathrm{g}}(t, \mathbf{p})^{T}\right)^{T}$ of the model accounting for the effects of the soil resistivity as

$$
y^{\mathrm{m}, \mathrm{g}}\left(\mathbf{p}, \rho, t_{k}\right)=G\left(z^{-1}, \rho\right) y^{\mathrm{m}, 0}\left(\mathbf{p}, t_{k}\right),
$$

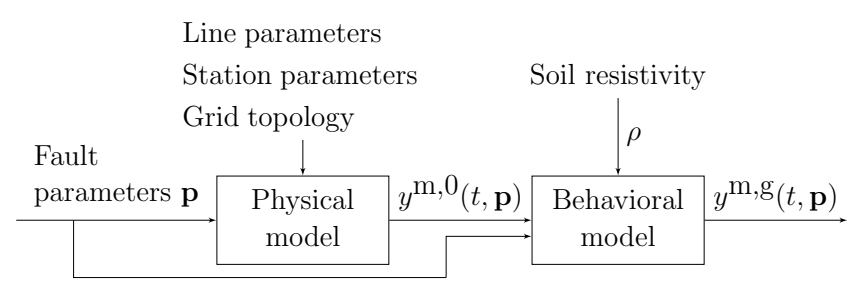

Fig. 5: Combined physical and behavioral model

where $G\left(z^{-1}\right)$ is a discrete time low pass filter tuned so that the output of the combined model fits the measurements obtained with an EMT simulation. As the effect of the soil resistivity varies with the fault distance, the ground filter depends on the fault parameters $\mathbf{p}$ through $d_{\mathrm{f}}$. Hence the combined model still depends explicitly on the fault parameters which value has to be identified.

\subsection{Fault identification}

Consider a fault is suspected at the relay at station $q$ monitoring line $e$ using an detection module such as an undervoltage, see [15]. Considering a fault actually occurred on the line $e$, the FID evaluates a ML estimate $\widehat{\mathbf{p}}$ of the vector of fault parameters $\mathbf{p}$ using the voltage and current measurements $\left(v_{q, e}(t), i_{q, e}(t)\right)$ and model $\left(v_{q, e}^{\mathrm{m}}(\mathbf{p}, t), i_{q, e}^{\mathrm{m}}(\mathbf{p}, t)\right)$. This amounts to minimizing a cost function defined as: [17]

$c^{(n)}=\lambda_{v}\left\|v_{q, e}^{\mathrm{m}}(\mathbf{p}, t)-v_{q, e}(t)\right\|_{2}^{2}+\lambda_{i}\left\|i_{q, e}^{\mathrm{m}}(\mathbf{p}, t)-i_{q, e}(t)\right\|_{2}^{2}$

where $\lambda_{v}, \lambda_{i}$ are the weights given to voltage and current, respectively. The amount of measurement points used is noted $n$. The FID evaluates iteratively an estimate of the fault parameters: the estimation algorithm is started as soon as an abnormal behavior is detected and the relay and the estimate $\widehat{\mathbf{p}}$ is updated when new measurements are available.

For each $\widehat{\mathbf{p}}$, an approximate confidence region $\mathcal{R}^{(\alpha)}(\widehat{\mathbf{p}})$ is evaluated. To determine whether the estimate is consistent with the hypothesis that the monitored line is faulty, two tests are considered. First, a validity test determines whether $\widehat{\mathbf{p}}$ is included in some domain of interest. The estimated parameters must belong to $\mathcal{D}_{\mathbf{p}}$ which represents plausible values for the fault parameters. $\mathcal{D}_{\mathbf{p}}$ may be defined as

$$
\mathcal{D}_{\mathbf{p}}=\left\{\left(d_{\mathrm{f}}, R_{\mathrm{f}}\right) \mid d_{\min } \leq d_{\mathrm{f}} \leq d_{\max }, R_{\min } \leq R_{\mathrm{f}}<R_{\max }\right\}
$$

where $\left(d_{\min }, d_{\max }\right)$ defines the portion of the line actually monitored by the relay and $\left(R_{\min }, R_{\max }\right)$ the range of fault resistance that requires fast decision, since a high value of $R_{\mathrm{f}}$ corresponds to a non-critical fault for which more time is available to take action, as investigated in Section 2.2. Typical boundary values are $d_{\min }=0 \mathrm{~km}, d_{\max }=90 \% d_{q q^{\prime}}$ and $R_{\min }=0 \Omega, R_{\max }=200 \Omega$. For faults occurring particularly far on the protected line $\left(d_{\mathrm{f}}>90 \% d_{q q^{\prime}}\right)$, the small distance between the fault and the remote relay at station $q^{\prime}$ protected the line allows to use inter-tripping. 
Second, an accuracy test determines whether the area of the $95 \%$ confidence region of the estimated parameters $\mathcal{R}^{(\alpha)}(\widehat{\mathbf{p}})$ goes under a threshold $t r_{95}$. This confidence region is computed based on the Fisher information matrix, assuming usual statistical properties such as normal independent distribution of the measurement noise, [17]. If both tests are satisfied, the fault is deemed to affect line $e$. When one is unable to conclude, the FID waits for the availability of $\Delta n$ additional measurements to update $\widehat{\mathbf{p}}$ and $\mathcal{R}^{(\alpha)}(\widehat{\mathbf{p}})$. Once enough measurements have been made available without allowing the FID to conclude, the fault is deemed to be located elsewhere in the grid, or to be non-existent.

\section{Simulation results}

This section presents simulation results of the identification algorithm. Measurements in case of faults are provided by EMTP-RV and the FID is implemented in Matlab. The parameter estimation algorithm is initialized at $\mathbf{p}_{0}=\left(R_{\text {init }}, d_{\text {init }}\right)=$ $(1 \Omega, 6 \mathrm{~km})$. The number of data points added after each iteration is fixed at $\Delta n=10$ by trial and error. The maximum measurement window that can be used to determine whether the line is faulty is set to $3 d / c_{\mathrm{w}}$ for a relay monitoring a line of length $d$ where waves propagate at a speed $c_{\mathrm{w}}$. One considers a pole-to-ground fault occurring at $t_{\mathrm{f}}=0$ in line $L_{14}$ of the grid in Figure 1 at a distance $d_{\mathrm{f}}^{*}=80 \mathrm{~km}$ from station 1 with an impedance of $R_{\mathrm{f}}^{*}=15 \Omega$ which represents a typical use case of the algorithm, see Section 2. Once an abnormal behavior is detected at relay $R_{14}$, the identification algorithm is started. Its behavior can be analyzed by plotting the contour of the cost function to be minimized at each iteration as well as the trajectory of the estimate $\left(d_{\mathrm{f}}, R_{\mathrm{f}}\right)$ of the fault parameter vector, see Figure 6 . The 95\% confidence ellipse of the estimated parameter vector is also displayed at each step. After each iteration, when new data points are taken into account, the argument of the minimum gets closer to $\left(d_{\mathrm{f}}^{*}, R_{\mathrm{f}}^{*}\right)$ and the cost contours concentrate around $\left(d_{\mathrm{f}}^{*}, R_{\mathrm{f}}^{*}\right)$. The estimate $\left(\widehat{d}_{\mathrm{f}}, \widehat{R_{\mathrm{f}}}\right)$ also gets closer to $\left(d_{\mathrm{f}}^{*}, R_{\mathrm{f}}^{*}\right)$ and the size of the confidence ellipsoid reduces. The estimation algorithm stops and correctly identifies the fault on the line after 13 iterations, requiring only measurements obtained in a time window of $136 \mu \mathrm{s}$. The estimated parameters are $\widehat{d}_{\mathrm{f}}=73 \mathrm{~km}$ and $\widehat{R}_{\mathrm{f}}=22 \Omega$ when the algorithm stops.

The voltage and current measurements simulated by the EMT software and the outputs of the parametric model for $\widehat{d}_{\mathrm{f}}=$ $73 \mathrm{~km}$ and $\widehat{R}_{\mathrm{f}}=22 \Omega$ are represented in Figure 7 . One sees that the observation of the first wave is enough for an accurate fault identification, though several waves can be used in case of a close fault. Hence, the amount of data necessary to identify faults is, to a large extent, independent of the fault distance on the protected line. Extensive simulations have been carried out on the fault cases presented in Section 2, showing that the amount of data necessary to identify faults occurring on the protected line is always less than $0.5 \mathrm{~ms}$. Since the algorithm can be started as soon as the first data are received at the
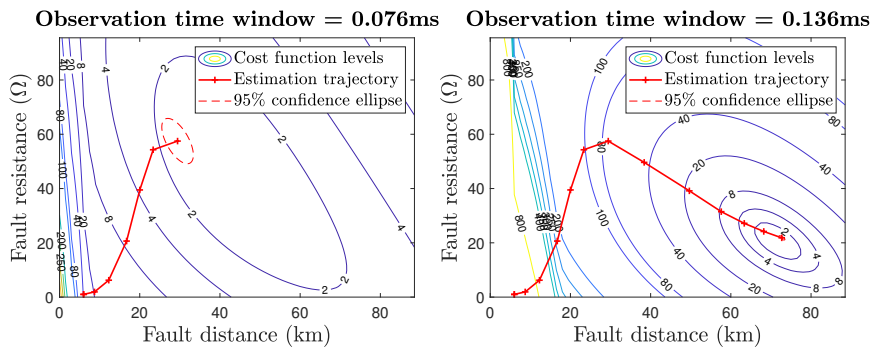

Fig. 6: Fault at $d_{\mathrm{f}}^{*}=80 \mathrm{~km}$ from station 3 (close fault) with an impedance of $R_{\mathrm{f}}^{*}=15 \Omega$ : evolution of the contour plot of the cost function and estimated parameters at iterations 6 and 13 .
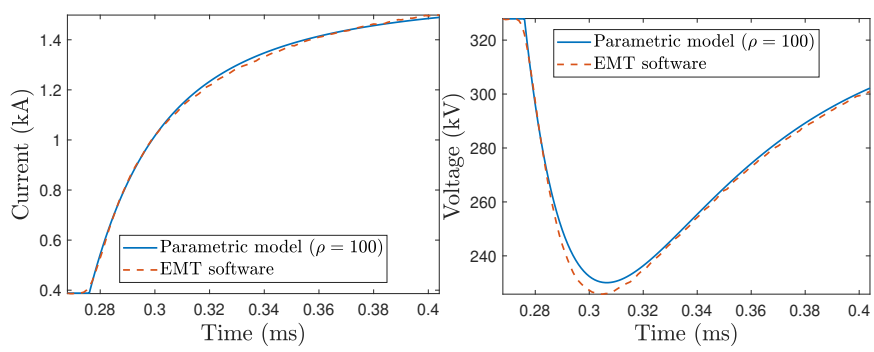

Fig. 7: Simulated current (left) and voltage (right) measurements compared to the combined model outputs for $\widehat{d_{\mathrm{f}}}=26 \mathrm{~km}$ and $\widehat{R}_{\mathrm{f}}=21 \Omega$.

relay, it is reasonable to consider, as in Section 2, that the total time to perform fault detection and identification, including data storage and processing, is about $0.5 \mathrm{~ms}$. This however assumes fast enough implementation and hardware for real-time application.

To analyze the behavior of the identification algorithm at the other relays, the evolution of the accuracy criterion at the 8 relays is plotted in Figure 8. Though the fault is rapidly identified at relays $R_{14}$ and $R_{41}$, the evolution of the accuracy criterion is plotted until the maximum measurement window is reached to be compared to the evolution at the other relays. Since the fault is close to station 1 , it is first detected at relays $R_{12}, R_{13}$ and $R_{14}$. As seen above, the latter identifies the fault as internal to its protected line, $L_{14}$, after using only $136 \mu \mathrm{s}$ of measurements. The accuracy criteria is never satisfied at relays $R_{12}$ and $R_{13}$, indicating that the fault is not on their respective line, $L_{12}$ and $L_{13}$. At $t=0.7 \mathrm{~ms}$ the fault is detected at the relays of station $4, R_{41}$ and $R_{42}$. At relay $R_{41}$, the fault is again correctly identified after few iterations whereas the algorithm at relay $R_{42}$ never satisfies the accuracy test. The fault is finally detected by the other relays at stations 3 and 1 where, as expected, none of the relays identify the fault to be on their respective protected line. This illustrates that the method is able to identify internal faults using very few measurements while rejecting faults occurring on neighboring lines. In particular, the algorithm is inherently directional as the expected direction of the current for a forward fault is included in the model. 


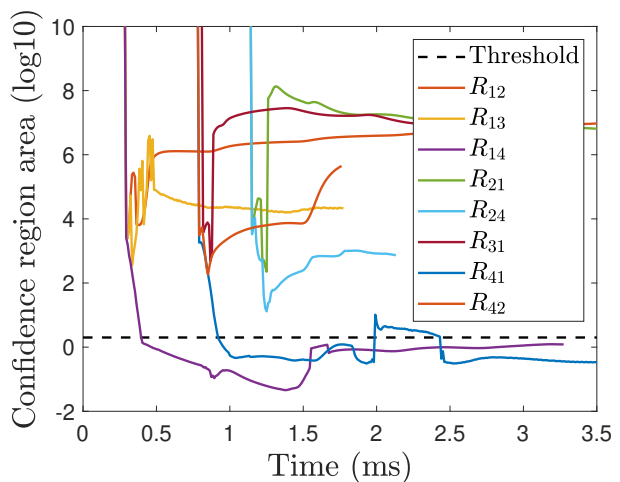

Fig. 8: Evolution of the accuracy criteria at the 8 relays.

\section{Conclusion}

This paper presents a novel single-ended algorithm for fault identification for meshed HVDC grids. A parametric model consisting of a physical part and a behavioral part is used to describe the voltage and current evolution just after the occurrence of a fault. Then, a maximum-likelihood estimate of the fault parameter is performed and updated each time new measurements are available. The confidence region associated to the parameter estimate is used to decide if the fault affects the protected line or if more measurements are needed. The approach is evaluated on a simulated four-node meshed grid. On the considered example, the current and voltage need to be observed during less than $0.2 \mathrm{~ms}$ to get an accurate estimate of the fault characteristics and to identify consistently the faulty line. A way to further improve the robustness and the precision of the proposed approach, one can run several estimation algorithms with different initialization within the same relay and rely on a voting system among the provided estimates.

\section{Acknowledgments}

This work was carried out at the SuperGrid Institute, an institute for the energetic transition (ITE). It is supported by the French government under the frame of "Investissements d'avenir" program with grant reference number ANE-ITE002-01

\section{References}

[1] E. Pierri, O. Binder, N. G. Hemdan, and M. Kurrat, "Challenges and opportunities for a European HVDC grid," Renewable and Sustainable Energy Reviews, vol. 70, pp. 427-456, 2017.

[2] P. Rodriguez and K. Rouzbehi, "Multi-terminal DC grids: challenges and prospects," Journal of Modern Power Systems and Clean Energy, vol. 5, no. 4, pp. 515523, 2017.

[3] WP4 PROMOTIoN, "Report on the broad comparison of protection philosophies for the identified grid topologies," Tech. Rep., 2018.
[4] R. Li, L. Xu, and L. Yao, "DC fault detection and location in meshed multiterminal HVDC systems based on DC reactor voltage change rate," IEEE Transactions on Power Delivery, vol. 32, no. 3, pp. 1516-1526, 2017.

[5] G. Auran, B. Raison, J. Descloux, and S. Nguefeu, "A novel pole-to-ground fault detection algorithm for meshed HVDC grids with half-bridge MMC converters and full recourse to DC circuit breakers," in Proc. 13th IET International Conference on Developments in Power System Protection (DPSP), 2016, pp. 1-6.

[6] A. Guzman-Casillas, B. Kasztenny, Y. Tong, and M. V. Mynam, "Accurate single-end fault locating using traveling-wave reflection information," in Proc. Developments In Power System Protection, Belfast, 2018, pp. $1-6$.

[7] J. Suonan, J. Zhang, Z. Jiao, L. Yang, and G. Song, "Distance Protection for HVDC Transmission Lines Considering Frequency-Dependent Parameters," IEEE Transactions on Power Delivery, vol. 28, no. 2, pp. 723732, 2013.

[8] Hermann W. Dommel, "Digital Computer Solution of Electromagnetic Transients in Single-and Multiphase Networks," IEEE Transactions on Power Apparatus and Systems, vol. 88, no. 4, pp. 388 - 399, 1969.

[9] K. Shinoda, A. Benchaib, J. Dai, and X. Guillaud, "Virtual Capacitor Control for Stability Improvement of HVDC System Comprising DC Reactors," in Proc. 15th IET International Conference on AC and DC Power Transmission, 2019, pp. 1-6.

[10] WP6 PROMOTIoN, "Develop system level model for hybrid DC CB,” Tech. Rep. 691714, 2016.

[11] G. Auran, "Full selective protection strategy for multiterminal cable HVDC grids based on HB-MMC converters," PhD Thesis, Université Grenoble Alpes, 2017.

[12] G. Li, J. Liang, C. E. Ugalde-loo, and P. Coventry, "Impacts of DC circuit breakers on AC/DC system stability subject to DC faults," Proc. 2nd International Conference on HVDC (HVDC 2016), no. June, 2018.

[13] H. Pang and X. Wei, "Research on Key Technology and Equipment for Zhangbei 500kV DC Grid," Proc. 2018 International Power Electronics Conference, IPEC-Niigata - ECCE Asia 2018, pp. 2343-2351, 2018.

[14] J. De Andrade Suárez and E. Sorrentino, "Typical expected values of the fault resistance in power systems," in Proc. IEEE/PES Transmission and Distribution Conference and Exposition: Latin America, T and D-LA 2010, 2010, pp. 602-609.

[15] F. Kong, Z. Hao, S. Zhang, and B. Zhang, "Development of a novel protection device for bipolar HVDC transmission lines," IEEE Transactions on Power Delivery, vol. 29, no. 5, pp. 2270-2278, 2014. 
[16] Allan Greenwood, Electrical Transients in Power Systems, 2nd ed., I. John Wiley \& Sons, Ed. John Wiley \& Sons, 1991.

[17] E. Walter and L. Pronzato, Identification of parametric models from experimental data. Springer-Verlag London, 1997. 